\title{
Universality and non-universality of the growth law
}

\author{
Qirun Wang ${ }^{1}$ and Jie Lin ${ }^{1,2}$ \\ ${ }^{1}$ Center for Quantitative Biology, Peking University, Beijing, China \\ ${ }^{2}$ Peking-Tsinghua Center for Life Sciences, Peking University, Beijing, China
}

(Dated: December 3, 2021)

\begin{abstract}
An approximately linear relationship between the fraction of ribosomal proteins in the proteome $\left(\phi_{R}\right)$ and the growth rate $(\mu)$ holds in proliferating cells when the nutrient quality changes, often referred to as a growth law. While a simple model assuming a constant translation speed of ribosomes without protein degradation can rationalize this growth law, real protein synthesis processes are more complex. This work proposes a general theoretical framework of protein synthesis, taking account of heterogeneous translation speeds among proteins and finite protein degradation. We introduce ribosome allocations as the fraction of active ribosomes producing certain proteins, with two correlation coefficients respectively quantifying the correlation between translation speeds and ribosome allocations, and between protein degradation rates and mass fractions. We prove that the growth law curve generally follows $\phi_{R}=\left(\mu+c_{1}\right) /\left(c_{2} \mu+c_{3}\right)$ where $c_{1}, c_{2}$, and $c_{3}$ are constants depending on the above correlation coefficients and the translation speed of ribosomal proteins. Our theoretical predictions of $\phi_{R}$ agree with existing data of Saccharomyces cerevisiae. We demonstrate that when different environments share similar correlation coefficients, the growth law curve is universal and up-bent relative to a linear line in slow-growth conditions, which appears valid for Escherichia coli. However, the growth law curve is non-universal and environmental-specific when the environments have significantly different correlation coefficients. Our theories allow us to estimate the translation speeds of ribosomal and non-ribosomal proteins based on the experimental growth law curves.
\end{abstract}

6 Cells can adapt to different environments and alter the 7 expression levels of multiple genes correspondingly. The 8 genome-wide gene expression profile can change signif9 icantly as cells switch between different environments. 10 However, proliferating cells, including bacteria and uni11 cellular eukaryotes, exhibit a simple growth law as the nu12 trient quality changes: an approximately linear relation 13 exists between the fraction of ribosomal proteins in the 14 proteome $\left(\phi_{R}\right)$ and the growth rates $(\mu), \phi_{R}=\mu / \kappa+\phi_{0}$ ${ }_{15}[1-6]$. This growth law can be rationalized by a sim16 ple translation model (STM): ribosomes are engaged in 17 translation with a constant translation speed that is pro18 portional to $\kappa[2,4]$. $\phi_{0}$ represents the fraction of inactive 19 ribosomes that are not producing proteins, independent 20 of environments in the STM. While the STM is simple 21 and intuitive, it appears to break down in slow-growth 22 conditions of Escherichia coli (doubling time longer than 23 60 mins at $37^{\circ} \mathrm{C}$ ) in which more ribosomes are produced 24 than the expectation from the STM [7].

${ }_{25}$ We note that there are two important biological fea${ }_{26}$ tures (if not all) beyond the STM, which, as we show in 27 this work, are crucial to interpret the experimental data ${ }_{28}$ of $\phi_{R}$ versus $\mu$ (the growth law curve). The first is the 29 heterogeneous translation speeds of ribosomes produc30 ing different proteins. Recent studies demonstrated that 31 the translation speeds are highly heterogeneous among 32 different proteins due to multiple mechanisms, including 33 codon usages [8] and amino acid compositions [9]. Be34 cause of the universalities of these mechanisms, one ex35 pects that heterogeneous translation speeds among pro36 teins are universal across different organisms. In par37 ticular, the translation speeds of ribosomal proteins are
38 significantly slower than the average translation speed 39 over non-ribosomal proteins due to the abundance of 40 positively charged amino acids on ribosomal proteins [9]. ${ }_{41}$ Nowadays, the ribosome profiling technique allows us to 42 quantify the allocation of ribosomes towards the produc43 tion of different proteins. These experimental techniques 44 enable us to rethink the growth law in the presence of 45 heterogeneity in translation speeds [9].

46 The second feature is finite protein degradation rates. ${ }_{47}$ The STM neglects protein degradation and predicts that 48 at zero growth rate, $\phi_{R}=\phi_{0}$ so that all ribosomes are 49 inactive. However, this contradicts with experiments of 50 nongrowing bacteria in which significant translation ac51 tivities are observed [10]. Protein degradation must be 52 considered at zero growth rate to balance protein pro53 duction to ensure a constant protein mass. Therefore, 54 protein degradation should be important to the growth 55 law, at least in slow-growth conditions.

56 In this work, we show that the heterogeneous transla57 tion speeds and finite protein degradations significantly 58 influence the growth law connecting the fraction of ri59 bosomal proteins and the growth rate when the nutrient ${ }_{60}$ quality changes. The fractions of ribosomal proteins $\phi_{R}$ ${ }_{61}$ are generally different in different environments, even if 62 they lead to the same growth rates. Besides the growth 63 rate, $\phi_{R}$ depends on two correlation coefficients among ${ }_{64}$ proteins. One is between the translation speeds and ribo65 some allocations, and the other is between the correlation ${ }_{66}$ coefficient between protein degradation rates and mass ${ }_{67}$ fractions. We compute the above correlation coefficients 68 using proteomics and ribosomal profiling datasets of $S$. 69 cerevisiae [11]. Interestingly, we find that the correlation 


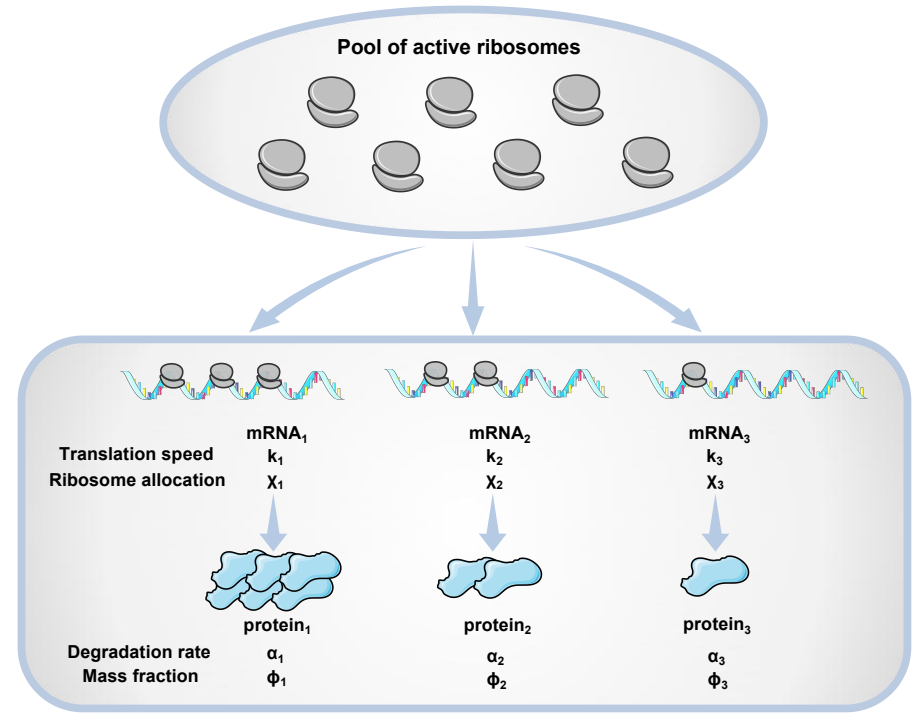

FIG. 1. Given a constant environment, cells actively allocate different fractions of active ribosomes $\left(\chi_{i}\right)$ to translate mRNAs corresponding to different proteins. In general, the translation speeds $k_{i}$ are heterogeneous among proteins. $\alpha_{i}$ is the degradation rate of protein $i . \chi_{i}, k_{i}$ and $\alpha_{i}$ together determine the mass fraction of protein $i$. The ribosome allocation strategies reflect the adaption of cells to different environments. In this schematic, we show three proteins for simplicity.

70 between the translation speed and ribosome allocations 99

71 become stronger when the growth rate decreases; namely, 72 cells tend to produce more proteins with higher transla- 100 73 tion speeds in poor nutrient. In contrast, the correlation 74 between the protein degradation rates and mass fractions 75 is almost independent of growth rates.

76 We derive the general form of growth law involving 77 the above correlations. We demonstrate that for envi78 ronments with similar correlation coefficients, the growth 79 law curve is universal and has the following form, $\phi_{R}=$ so $\left(\mu+c_{1}\right) /\left(c_{2} \mu+c_{3}\right)$ where $c_{1}, c_{2}$, and $c_{3}$ are constants ${ }_{81}$ depending on the above correlation coefficients and the 82 translation speed of ribosomal proteins. We prove that 83 the growth law curve must be monotonically increasing 84 and convex, which justifies the upward bending of the 85 growth law curve of $E$. coli observed in slow-growth con${ }_{86}$ ditions relative to a linear line [7]. However, if the exper87 iments are implemented in multiple environments with 88 dramatically different correlation coefficients, the growth 89 law curve is generally non-universal and environmental90 specific. Our analysis of experimental data suggests that ${ }_{91}$ this scenario may apply to $S$. cerevisiae. Our theories al${ }_{92}$ low us to fit the experimentally measured growth law ${ }_{93}$ curves by our model predictions, from which we can 94 estimate the translation speed of ribosomal and non95 ribosomal proteins. Consistent with direct experimen96 tal measurements [9], the estimated translation speed ${ }_{97}$ of ribosomal proteins is indeed much slower than non98 ribosomal proteins.

\section{RESULTS}

\section{Model of protein synthesis}

101 Given a constant environment, we consider a popula102 tion of cells with a constant growth rate, and the protein ${ }_{103}$ synthesis processes are in a steady state. Ribosome pro104 filing allows us to quantify the fraction of ribosomes in 105 the pool of total active ribosomes producing protein $i$, 106 which we call ribosome allocation $\chi_{i}$. Here the index ${ }_{107} i$ represents one particular protein $i$. Mass spectrome${ }_{108}$ try also allows us to measure the mass fractions $\phi_{i}$ of 109 all proteins in the proteome [12]. The elongation rate 110 of ribosomes on the corresponding mRNAs is $v_{i}$, which 111 is the number of translated amino acids per unit time. ${ }_{112}$ Note that $v_{i}$ is the averaged elongation rate over the se${ }_{113}$ quence of the corresponding mRNA so that each protein 114 has one $v_{i}$. We also assume that protein $i$ degrades with ${ }_{115}$ a constant rate $\alpha_{i}$. The mass production rate of protein ${ }_{116} i$ becomes

$$
\frac{d M_{i}}{d t}=v_{i} a_{i} \chi_{i}\left(R-R_{0}\right)-\alpha_{i} M_{i} .
$$

${ }_{117}$ Here $R$ is the number of ribosomes, and $R_{0}$ is the number 118 of inactive ribosomes. $a_{i}$ is the averaged mass of amino 119 acids over the sequence of protein $i$. In the following anal120 ysis, we define $k_{i}=v_{i} a_{i}$ as the amino acid mass-weighted 121 translation speed and denote it as the translation speed 122 for simplicity. Our model is summarized in Figure 1. ${ }_{123}$ Recently, Dai et al. showed that for E. coli the trans124 lation speeds of many proteins decrease as the growth 125 rate decreases, but maintain finite values at zero growth 
126 rate [7]. They proposed a model in which the translation ${ }_{179}$ expression of $\phi_{R}$ as (see detailed derivations in Appendix ${ }_{127}$ speeds are the same for all proteins and depend on the ${ }_{180} \mathrm{~B}$ )

${ }_{128}$ ribosomal fraction $\phi_{R}$ in a Michaelis-Menten way, consis129 tent with their experimental data. However, their model 130 predicts a downward bending of the growth law curve 131 in slow-growth conditions relative to a linear line, in con132 trast to the upward bending observed experimentally. To 133 reconcile the conflict, they proposed that the fraction of ${ }_{134}$ inactive ribosomes $\phi_{0}$ increases as the growth rate de135 creases, generating the upward bending of the growth 136 law curve. However, as far as we know, there is no di${ }_{137}$ rect experimental evidence supporting a larger fraction of ${ }_{138}$ inactive ribosomes $\phi_{0}$ in slow-growth conditions than in 139 fast-growth conditions. Interestingly, no noticeable bend140 ing is observed in the growth law curve of $S$. cerevisiae ${ }_{141}[6]$, suggesting that the upward bending of the growth ${ }_{142}$ law curve in slow-growth conditions may not be universal ${ }_{143}$ across organisms, consistent with our theoretical predic144 tions as we show later.

${ }_{145}$ We remark that a growth-rate dependent translation ${ }_{146}$ speed is undoubtedly a mechanism that the STM breaks ${ }_{147}$ down. However, in this work, we focus on the effects of ${ }_{148}$ heterogeneous translation speeds $k_{i}$ and finite degrada${ }_{149}$ tion rates $\alpha_{i}$. Therefore, we assume them to be invariant 150 of environments. We also mainly consider the effects of ${ }_{151}$ nutrient quality and do not consider the impact of an152 tibiotics in this work, which can decrease the overall ef153 fective translation speed and increase $\phi_{R}$ as the growth 154 rate decreases [4]. Thanks to the simplicity of our pro155 tein synthesis model, it can be analytically solved, and 156 the predictions are intriguing as we show later.

${ }_{157}$ We define the total protein mass $M=\sum_{i} M_{i}$, and the ${ }_{158}$ protein mass fraction $\phi_{i}=M_{i} / M$. Using Eq. (1), we 159 find the values of $\phi_{i}$ in the steady state as (see detailed 160 derivations in Appendix A)

$$
\phi_{i}=\frac{k_{i} \chi_{i}\left(\phi_{R}-\phi_{0}\right)}{m_{R}\left(\mu+\alpha_{i}\right)}
$$

${ }_{161}$ Here $\mu$ is the growth rate of the total protein mass ${ }_{162} \mu=\dot{M} / M$, and $m_{R}$ is the total amino acid mass of a 163 single ribosome. Since all proteins grow in the same rate ${ }_{164}$ in the steady-state, the growth rates of protein $i$ defined 165 as $\mu_{i}=\dot{M}_{i} / M_{i}=k_{i} \chi_{i}\left(\phi_{R}-\phi_{0}\right) /\left(m_{R} \phi_{i}\right)-\alpha_{i}$ must be ${ }_{166}$ equal to $\mu$, which can be easily verified using Eq. (2). In 167 the following, $i=1$ is reserved for ribosomal proteins so ${ }_{168}$ that $\phi_{1}=\phi_{R}$ and $\mu_{1}=\mu_{R}=k_{R} \chi_{R}\left(1-\phi_{0} / \phi_{R}\right) / m_{R}-\alpha_{R}$. ${ }_{169}$ Here, $k_{R}$ and $\alpha_{R}$ are the effective translation speed, and ${ }_{170}$ degradation rate of the coarse-grained ribosomal protein 171 averaged over all ribosomal proteins. They are approxi172 mately independent of environments due to the tight reg173 ulation of relative doses of different ribosomal proteins 174 [13] and their generally low degradation rates.

${ }_{175}$ Given the ribosome allocations $\chi_{i}$, the protein degra${ }_{176}$ dation rates $\alpha_{i}$ and the translation speeds $k_{i}$, one obtains 177 a unique solution of $\phi_{i}$ and $\mu$. We can express the growth ${ }_{213}$ Here, the bracket represents an average over all non178 rate as $\mu=\sum_{i} \phi_{i} \mu_{i}$ and rewrite Eq. (2) to obtain the 214 ribosomal proteins. Because the ribosomal allocations $\chi_{i}$

$$
\begin{aligned}
& a=\frac{k_{R}-\langle k\rangle_{\chi}}{k_{R}\left(1-\phi_{0}\right)+\langle k\rangle_{\chi} \phi_{0}}, \\
& b=\frac{k_{R}\langle k\rangle_{\chi}}{\left.m_{R}\left[k_{R}\left(1-\phi_{0}\right)+\langle k\rangle_{\chi} \phi_{0}\right)\right]} .
\end{aligned}
$$

200 We are particularly interested in the sign of $a$ because it 201 determines the shape of the $\phi_{R}(\mu)$ curve. If $k_{R}$ is smaller 202 than $\langle k\rangle_{\chi}, a$ is negative so that the second derivative of 203 the $\phi_{R}(\mu)$ curve is positive. In other words, the $\phi_{R}(\mu)$ 204 curve is upward bent in slow-growth conditions.

$\langle k\rangle_{\chi}$ depends on both the elongation speeds $k_{i}$ and the 206 ribosome allocations $\chi_{i}$. To find its value, we further ${ }^{207}$ rewrite $\langle k\rangle_{\chi}=\langle k\rangle\left(1+I_{\chi, k}\right)$. Here $\langle k\rangle$ is the arithmetic 208 average of translation speeds over all non-ribosomal pro209 teins, which is constant and independent of environ210 ments. $I_{\chi, k}$ is a metric we use to quantify the correla${ }_{21}$ tion between the ribosome allocations and the translation 212 speeds:

$$
I_{\chi, k}=\frac{\left\langle\widetilde{\chi}_{i} k_{i}\right\rangle-\left\langle\widetilde{\chi}_{i}\right\rangle\langle k\rangle}{\left\langle\widetilde{\chi}_{i}\right\rangle\langle k\rangle} .
$$

181 Here, $\phi_{0}$ is the mass fraction of inactive ribosomes, which 183 find that if all proteins have the same translation speed ${ }_{184}\left(k_{i}=k\right.$ for all $\left.i\right)$ and protein degradations are negligible ${ }_{185}\left(\alpha_{i}=0\right)$, Eq. (3) is reduced to the STM result.

\section{$186 \quad$ Effects of heterogeneous translation speeds} 188 translation speeds and degradation rates, we choose to 189 study them separately. Therefore, we first simplify the 190 model by taking $\alpha_{i}=0$ for all proteins and only con191 sider the effects of heterogeneous translation speeds $k_{i}$. 193 Eq. (3). Here, $N$ is the number of genes and $\chi_{i}=$ ${ }_{194}\left(1-\chi_{R}\right) \tilde{\chi}_{i}$ so that $\sum_{i=2}^{N} \tilde{\chi}_{i}=1 . k_{R}$ is the translation 195 speed of ribosomal proteins. In the following, we define ${ }_{197}\langle k\rangle_{\chi}=\sum_{i=2} k_{i} \widetilde{\chi}_{i}$ as the $\chi$-weighted average translation
oll non-ribosomal proteins. As we derive in 198 Appendix $\mathrm{C}$, the fraction of ribosomal proteins can be 199 written exactly as a Hill function of the growth rate:

where 4) 

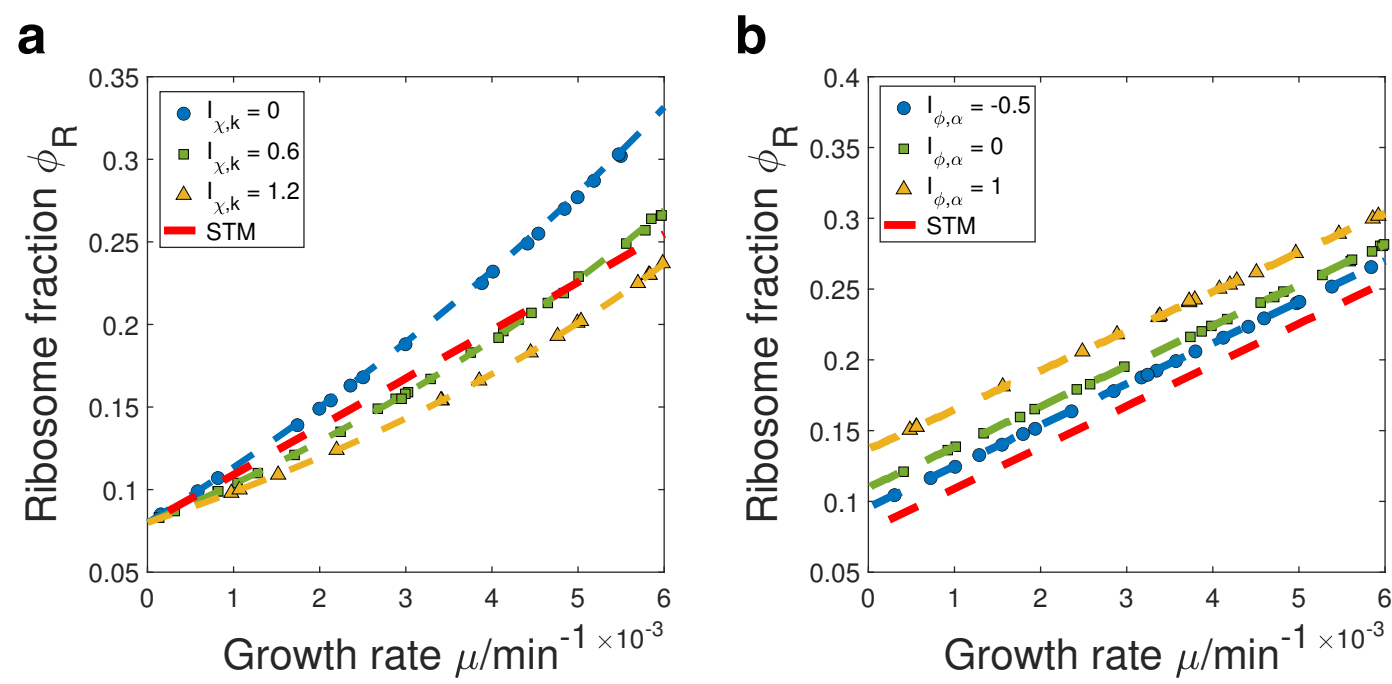

FIG. 2. Numerical simulations of the growth law curves. (a) We simulate the case of heterogeneous translation speeds and compare our numerical simulations with model predictions (dashed lines). Each data point has its own randomly sampled $\chi_{i}$ and we show the results with preselected $I_{\chi, k}$ values. The red dash line represents the predictions of the STM in which all proteins have the same translation speed $\langle k\rangle$. (b) Same analysis in which we simulate the case of finite protein degradation rates.

215 are generally different in different environments, we use ${ }_{216} I_{\chi, k}$ to characterize an environment. Imagine that we ${ }_{217}$ grow cells in multiple environments with equal $I_{\chi, k}$. We 218 find that as long as $I_{\chi, k}$ is not too close to -1 , which we 219 confirm later using experimental data, $a$ is always nega220 tive since the translation speed of ribosomal proteins $k_{R}$ ${ }_{221}$ is much lower than $\langle k\rangle[9]$. Therefore, Eq. (4) predicts 222 an upward bending of the $\phi_{R}(\mu)$ curve in slow-growth 223 conditions.

${ }_{224}$ We verify the above theoretical predictions by numer225 ically simulating the model of protein synthesis (Ap${ }_{226}$ pendix E). The translation speeds are randomly sam${ }_{227}$ pled among proteins and fixed for all environments, with ${ }_{228} k_{R}<\langle k\rangle$. We randomly sample $\chi_{i}$ for each environment 229 and compute the resulting growth rate $\mu$ and protein 230 mass fractions $\phi_{i}$. We show the results from environ${ }_{231}$ ments with preselected $I_{\chi, k}$, which agree well with the ${ }_{232}$ theoretical formula Eq. (4) (Figure 2a).

\section{$233 \quad$ Effects of finite protein degradation rates}

${ }^{234}$ We now discuss the effects of finite protein degrada235 tion rates. For simplicity, we assume that the transla236 tion speeds are homogeneous and equal to $k$ for all pro${ }_{237}$ teins. We rewrite the $\sum_{i} \alpha_{i} \phi_{i}$ term in Eq. (3) such ${ }_{238}$ that $\sum_{i} \alpha_{i} \phi_{i}=\alpha_{R} \phi_{R}+\left(1-\phi_{R}\right) \sum_{i=2}^{N} \alpha_{i} \widetilde{\phi}_{i}$. Here, ${ }_{239} \phi_{i}=\left(1-\phi_{R}\right) \widetilde{\phi}_{i}$ so that $\sum_{i=2}^{N} \widetilde{\phi}_{i}=1$. We define 240 the $\phi$-averaged degradation rates over all non-ribosomal ${ }_{241}$ proteins as $\langle\alpha\rangle_{\phi}=\sum_{i=2}^{N} \alpha_{i} \widetilde{\phi}_{i}$. Therefore, Eq. (3) can be 242 written as

$$
\phi_{R}=\frac{\mu+c}{k / m_{R}+d}+\phi_{0}
$$

where

$$
\begin{aligned}
& c=\langle\alpha\rangle_{\phi}\left(1-\phi_{0}\right)+\alpha_{R} \phi_{0}, \\
& d=\langle\alpha\rangle_{\phi}-\alpha_{R} .
\end{aligned}
$$

${ }_{243}$ To find the sign of $d$, we further rewrite $\langle\alpha\rangle_{\phi}$ as $\langle\alpha\rangle_{\phi}=$ ${ }_{244}\langle\alpha\rangle\left(1+I_{\phi, \alpha}\right)$ where $\langle\alpha\rangle$ is the arithmetic average of degra245 dation rates over all non-ribosomal proteins. $I_{\phi, \alpha}$ is a 246 metric we use to characterize an environment by quanti${ }_{247}$ fying the correlation between the protein mass fractions 248 and degradation rates:

$$
I_{\phi, \alpha}=\frac{\left\langle\widetilde{\phi}_{i} \alpha_{i}\right\rangle-\left\langle\widetilde{\phi}_{i}\right\rangle\langle\alpha\rangle}{\left\langle\widetilde{\phi}_{i}\right\rangle\langle\alpha\rangle} .
$$

${ }_{249}$ Here, the bracket represents an average over all non250 ribosomal proteins.

251 Imagine that we grow cells in multiple environments ${ }_{252}$ with equal $I_{\phi, \alpha}$. We assume that the degradation rate ${ }^{253}$ of ribosomal protein $\alpha_{R}$ is slower than the average of 254 non-ribosomal proteins $\langle\alpha\rangle$, which is biologically reason255 able since ribosomal proteins are generally non-degraded. ${ }_{256}$ Therefore, as long as $I_{\phi, \alpha}$ is not too close to -1 , which we ${ }_{257}$ confirm later using experimental data, $d$ is positive since ${ }_{258} \alpha_{R}$ is always smaller than $\langle\alpha\rangle_{\phi}$. Therefore, our model 259 predicts that the growth law curve is linear given a con260 stant $I_{\phi, \alpha}$ and finite protein degradation decreases the 261 slope relative to the STM. The intercept at $\mu=0$ is also 
262 larger than $\phi_{0}$. Therefore, a finite fraction of ribosomes 309 263 are still actively translating at zero growth rate. We ver264 ify the above theoretical predictions by numerically sim265 ulations and randomly sample the protein degradation 266 rates that are fixed for all environments, with $\alpha_{R}<\langle\alpha\rangle$ 267 satisfied. We show the results from environments with 268 preselected $I_{\phi, \alpha}$ and our theoretical predictions Eq. (8) 269 are nicely confirmed (Figure $2 \mathrm{~b}$ ).

\section{The full model}

271 We now consider the full model with both the hetero272 geneities in the translation speeds and protein degrada273 tion rates. We find that the growth law curve has the 274 following general form,

$$
\phi_{R}=\frac{\mu+c_{1}}{c_{2} \mu+c_{3}}
$$

${ }_{275}$ where the expression of the constants, $c_{1}, c_{2}$ and $c_{3}$ are 276 shown in Appendix D. We prove that given fixed $I_{\chi, k}$ and ${ }_{277} I_{\phi, \alpha}$ (as long as they are not too close to -1 ), the growth 278 law curve must be monotonically increasing and convex, 279 which suggests an upward bending in slow-growth condi280 tions (Appendix D). The simulation results again match 281 well with the theoretical predictions (Figure 3a).

${ }_{282}$ In real situations, we remark that the actual growth 283 curve shape depends on the particular environments. To 284 verify this, we compute the resulting growth law curve 285 with multiple environments, and the $I_{\chi, k}$ and $I_{\phi, \alpha}$ of 286 each environment are randomly sampled from Gaussian 287 distributions (Figure $3 \mathrm{~b}$ and e) (Appendix E). We find 288 that when the Gaussian distributions have large standard 289 deviations, the growth law curve is non-universal and de290 pends on the particular chosen environments (Figure 3c). 291 This means that if we randomly pick some environments 292 from Figure 3c, the resulting growth law curves are gen- 346 293 erally different. In contrast, when the Gaussian distri294 butions have small standard deviations, the growth law 340 295 curve is well captured by our theoretical predictions Eq. 296 (12), because the environments share similar $I_{\chi, k}$ and ${ }_{350}$ ${ }_{297} I_{\phi, \alpha}$ (Figure 3f).

${ }_{298}$ To quantify the effects of heterogeneous $I_{\chi, k}$ and $I_{\phi, \alpha}$ 299 across environments, we repeatedly sample 20 random 300 points from Figure 3c, f and fit them using Eq.(12) (Ap301 pendix E). We find that when the chosen environments 302 have significantly different $I_{\chi, k}$ and $I_{\phi, \alpha}$, the median 303 root mean squared error RMSE $=1.69 \times 10^{-2}$ (Figure $3043 \mathrm{~d}$ ). In contrast, in the case of similar environments, ${ }_{305} \mathrm{RMSE}=4.44 \times 10^{-3}$ (Figure $3 \mathrm{~g}$ ). The above results 306 suggest that we can use the fitting error as a criterion of
307 the universality of the growth law curve, which we apply 308 to the experimental data later.

\section{Experimental tests of theories}

310 In this section, we test our model using published 311 datasets of $S$. cerevisiae [14] (Appendix F). For each 312 strain and nutrient quality, we computed the correla313 tion coefficients between the translation speeds and ri314 bosome allocations $I_{\chi, k}$, and the correlation coefficients 315 between the protein degradation rates and protein mass ${ }_{316}$ fractions $I_{\phi, \alpha}$. Given the values of $\mu, I_{\chi, k}$, and $I_{\phi, \alpha}$, 317 we predicted the fraction of ribosomal proteins $\phi_{R}$ using ${ }_{318} \mathrm{Eq}$. (12) (Figure 4a and e). We note that there is one 319 parameter $\phi_{0}$ that is not known experimentally. Inter320 estingly, by choosing a common $\phi_{0}=0.048$, our model 321 predictions nicely match the experimental measured val322 ues of $\phi_{R}$ (with one data point slightly above the theo323 retical prediction). We find that regardless of the data 324 processing procedures, the relative relationships between 325 the predicted curves always agree with that of the exper326 imental values (Appendix F and Supplementary Figure $327 \mathrm{~S} 1)$.

328 Our model is simplified as we assume that the trans329 lation speeds and protein degradation rates do not de330 pend on environments. Remarkably, our model predic331 tions still quantitatively match the experimental obser332 vations, suggesting that our assumptions may be good $3 з 3$ approximations for most situations. While our model 334 cannot predict the growth rate dependence of $\phi_{0}$, our re335 sults show that a constant fraction of inactive ribosomes 336 is consistent with existing datasets of $S$. cerevisiae.

Interestingly, we found that $I_{\phi, \alpha} \approx-0.33$ for all the 338 conditions we computed. However, $I_{\chi, k}$ are negatively 339 correlated with the growth rates, suggesting cells tend 340 to allocate more ribosomes to translate mRNAs with ${ }_{341}$ higher $k_{i}$ in poor nutrient conditions (Figure $4 \mathrm{~b}$ ). To 342 find out what genes acquire more resources when the en343 vironment is shifted, we perform Gene Set Enrichment 344 Analysis (GSEA) [15, 16] for wide type cells (Appendix $345 \mathrm{~F}$ ) and find that 8 gene sets from the Gene ontology ${ }_{346}$ (GO) $[17,18]$ database are enriched in both the GSEA 347 where genes are ordered by $k_{i}$ (denoted as $k_{i}$-ordered ${ }_{348}$ GSEA) and the GSEA where genes are ordered by $\log _{2}$ ${ }_{349}$ fold change $\left(\log _{2} \mathrm{FC}\right)$ of $\chi_{i}$ (denoted as $\log _{2} \mathrm{FC}$-ordered 350 GSEA) (Figure 4d).

351 We find that five gene sets related to stress response 352 are enriched in the regime of higher $k_{i}$ and increasing $353 \chi_{i}$ when the environment is changed from $2 \%$ glucose to $3542 \%$ glycerol (Figure 4c). This is consistent with the en355 vironmental stress response (ESR) of $S$. cerevisiae as an 356 adaptation to the shifts of environments [19]. We propose 357 that higher translation speeds of stress response genes en358 able cells to respond rapidly to environmental changes, 359 which is evolutionarily advantageous. We also find two 360 gene sets related to the rRNA process are enriched in the ${ }_{361}$ regime of lower $k_{i}$ and decreasing $\chi_{i}$ (Figure $4 \mathrm{c}$ ). This is 362 consistent with the lower $\phi_{R}$ in slow-growth conditions 

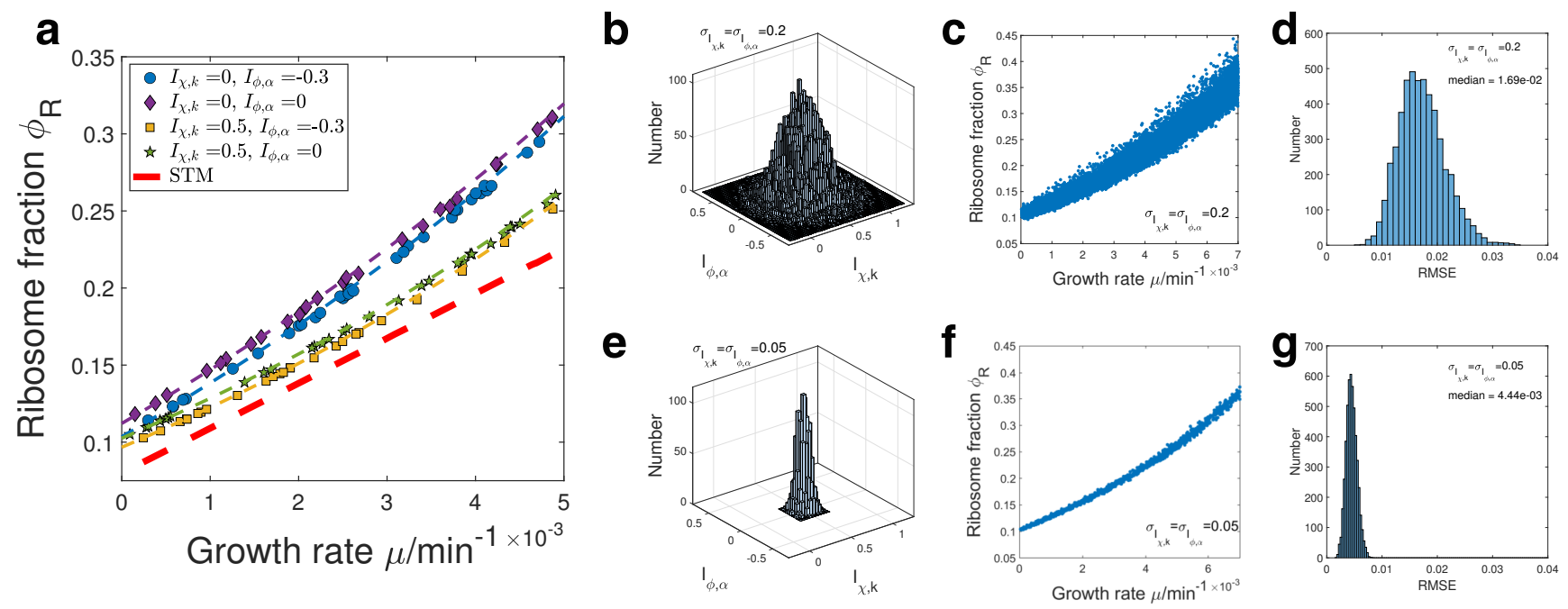

FIG. 3. Numerical simulations of the growth law curves with both heterogeneous translation speeds and protein degradation rates. (a) Numerical simulations with preselected $I_{\chi, k}$ and $I_{\phi, \alpha}$. The red dashed line is the prediction of the STM and other dashed lines represent our model predictions. (b) and (e) Two-dimensional Gaussian distribution of randomly sampled $I_{\chi, k}$ and $I_{\phi, \alpha}$. The mean of $I_{\chi, k}$ is 0.5 and the mean of $I_{\phi, \alpha}$ is 0 . The standard deviations $\sigma$ are indicated in the legends. (c) and (f) The resulting growth law curve where each point has randomly sampled $I_{\chi, k}$ and $I_{\phi, \alpha}$ from (b) and (e). (d) and (g) The distributions of the fitting RMSE corresponding to randomly chosen points in (c) and (f).

${ }_{363}$ (Figure 4a). We also perform GSEA for $\triangle$ Naa10 cells 364 and get similar results (Supplementary Figure S2).

366 An important application of our theories is that one 367 can estimate the translation speeds by fitting the exper368 imental growth law curve to our model prediction Eq. 369 (12) (Appendix G). Because there are 6 unknown param370 eters in the definition of $c_{1}, c_{2}$, and $c_{3}$ (Eq. (23-25)), we ${ }_{371}$ can estimate 3 of the parameters given the values of the 372 other 3 . For the $S$. cerevisiae data from Ref. [6], we use 373 the experimentally measured degradation rate of riboso374 mal proteins $\alpha_{R}$ and the mass of ribosomal proteins $m_{R}$ 375 as given. We approximate the $\phi$-averaged degradation ${ }_{376}$ rate $\langle\alpha\rangle_{\phi}$ by $\langle\alpha\rangle\left(1+I_{\phi, \alpha}\right)$ where $I_{\phi, \alpha}=-0.33$, and this is ${ }^{405}$ 377 justified by the observations that $I_{\phi, \alpha}$ is largely indepen378 dent of environments (Figure 4a). We find that the fitted 379 parameters $c_{1}, c_{2}$ and $c_{3}$ having a wide range of $95 \%$ con380 fidence intervals (Figure 5a) with RMSE $=1.35 \times 10^{-2}$, 381 which suggests that the growth law curve is non-universal 382 according to our simulations (Figure $3 \mathrm{~d}$ ). Indeed, the in${ }_{383}$ ferred values of $\phi_{0}, k_{R}$ and $\langle k\rangle_{\chi}$ have very large error bars ${ }_{384}$ (Figure $5 \mathrm{c}$ ). We also just fit the $\mathrm{C}$-limiting data points in ${ }_{385}$ Figure 5a [6] and obtain similar results (Supplementary 386 Figure S3).

${ }_{387}$ We also apply our theories to E. coli [7] (Figure 5b). ${ }_{388}$ Because most proteins are non-degradable in bacteria $389[20,21]$, we set $\alpha_{R}$ and $\langle\alpha\rangle_{\phi}$ as 0 , and the mass of ribo390 somal protein $m_{R}=8.07 \times 10^{5} \mathrm{Da}$ [12]. In this case, the
391 fitted parameters have much smaller range of $95 \%$ confi392 dence intervals with $\mathrm{RMSE}=3.60 \times 10^{-3}$. The estimated ${ }_{393} k_{R}$, and $\langle k\rangle$ are consistent with previous studies [22-24] 394 (Figure 5c). Our analysis of experimental data demon395 strates that the translation speed of ribosomal proteins 396 is indeed smaller than the $\chi$-averaged translation speed, 397 in agreement with experimental observations [9]. Our re398 sults suggest that $E$. coli has similar values of $I_{\chi, k}$ and $399 I_{\phi, \alpha}$ in the chosen environments of Ref. [7] so that it has 400 a universal growth law curve. In contrast, $S$. cerevisiae 401 appears to have significantly different $I_{\chi, k}$ and $I_{\phi, \alpha}$ across 402 different environments of Ref. [6] so that the growth law ${ }_{403}$ curve depends on the chosen environments and therefore 404 non-universal.

\section{Discussion}

In this work, we go beyond the simple translation 407 model and take account of the heterogeneous transla408 tion speeds and finite protein degradation. Given the 409 translation speeds and protein degradation rates, our 410 model is completely general and virtually applies to any 411 cells, including both proliferating cells $(\mu>0)$ and non${ }_{412}$ proliferating cells $(\mu=0)$. In this work, we mainly con${ }_{413}$ sider the scenario in which the growth rate changes due ${ }_{414}$ to the nutrient quality and the fraction of ribosomal pro415 teins $\left(\phi_{R}\right)$ increases monotonically as the growth rate 416 increases.

We demonstrate that the growth law curve is, in gen${ }_{418} \mathrm{eral}$, nonlinear and has the form Eq. (12). In particu- 
bioRxiv preprint doi: https://doi.org/10.1101/2021.12.02.471021; this version posted December 3, 2021. The copyright holder for this preprint (which was not certified by peer review) is the author/funder, who has granted bioRxiv a license to display the preprint in perpetuity. It is made available under aCC-BY-NC-ND 4.0 International license.

a

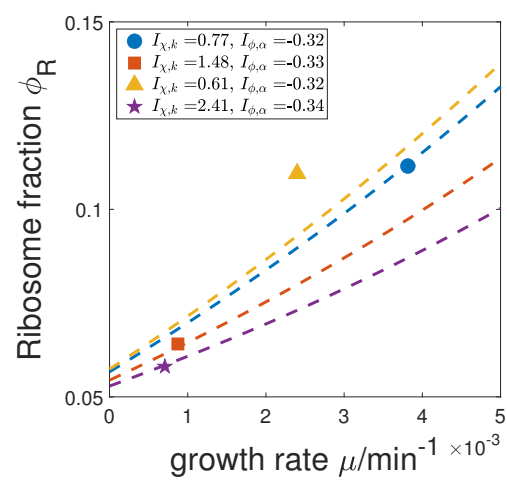

b

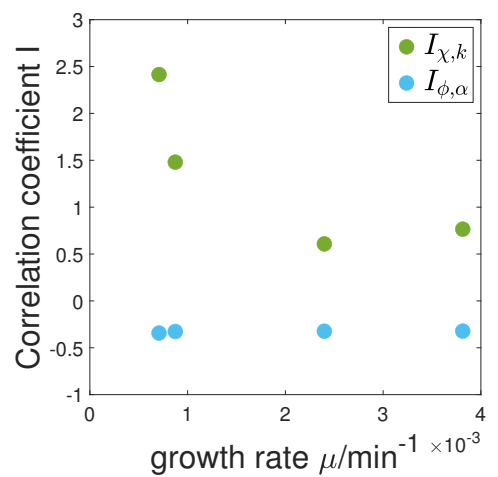

C

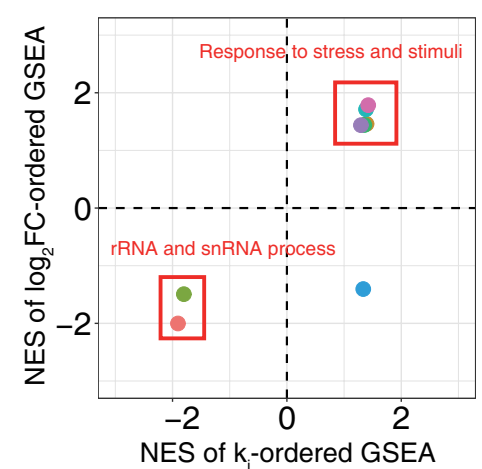

d

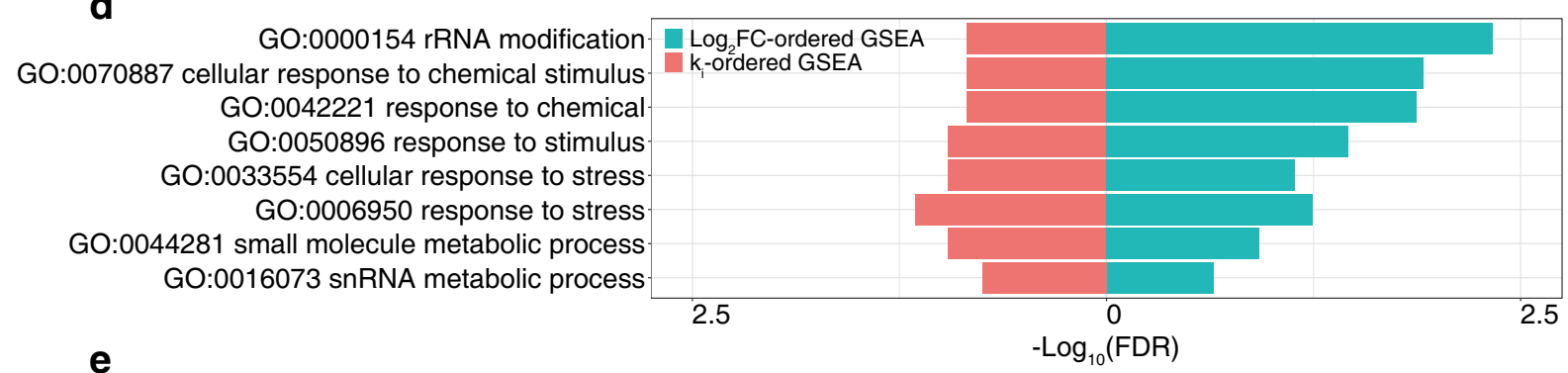

e

\begin{tabular}{|c|c|c|c|c|c|c|c|c|c|c|c|c|c|}
\hline Symble & Organism & Strain & Medium & $I_{x, k}$ & $I_{\phi, \alpha}$ & $\phi_{R}$ & $\underset{(1 / \mathrm{min})}{\mu}$ & $\begin{array}{c}\text { Effective } m_{R} \\
\text { (Da) }\end{array}$ & $\begin{array}{c}k_{R} \\
(\mathrm{Da} / \mathrm{min})\end{array}$ & $\begin{array}{c}<k> \\
(\mathrm{Da} / \mathrm{min})\end{array}$ & \begin{tabular}{|c|}
$\alpha_{R}$ \\
$(1 / \mathrm{min})$
\end{tabular} & \begin{tabular}{|c|}
$<\alpha>$ \\
$(1 / \mathrm{min})$
\end{tabular} & $\phi_{0}$ \\
\hline Reference & \multicolumn{8}{|c|}{$\begin{array}{l}\text { Friedrich et al., } \\
2021\end{array}$} & \multicolumn{2}{|c|}{$\begin{array}{c}\text { Riba et al., } \\
2019\end{array}$} & \multicolumn{2}{|c|}{$\begin{array}{c}\text { Lahtvee et al., } \\
2017\end{array}$} & - \\
\hline & \multirow{4}{*}{$\begin{array}{c}S . \\
\text { cerevisiae }\end{array}$} & BY4741 & $\mathrm{SC}+2 \% \mathrm{glu}$ & 0.77 & -0.32 & 0.11 & $3.81 \mathrm{e}-3$ & \multirow{4}{*}{$9.01 \mathrm{e} 5$} & \multirow{4}{*}{$2.07 \mathrm{e} 4$} & \multirow{4}{*}{$4.80 \mathrm{e} 4$} & \multirow{4}{*}{$4.83 e-4$} & \multirow{4}{*}{$1.10 \mathrm{e}-3$} & \multirow{4}{*}{$4.80 \mathrm{e}-2$} \\
\hline & & BY4741 & $\mid \mathrm{SC}+2 \%$ gly & 1.48 & -0.33 & 0.06 & $|8.74 \mathrm{e}-4|$ & & & & & & \\
\hline & & $\begin{array}{l}\text { BY4741 } \\
\Delta \mathrm{Naa} 10\end{array}$ & $\mathrm{SC}+2 \% \mathrm{glu}$ & 0.61 & -0.32 & 0.11 & $2.40 \mathrm{e}-3$ & & & & & & \\
\hline & & $\begin{array}{l}\text { BY4741 } \\
\Delta \mathrm{Naa} 10\end{array}$ & SC+2\%gly & 2.41 & -0.34 & 0.06 & 7.08e-4 & & & & & & \\
\hline
\end{tabular}

FIG. 4. Experimental analysis and theoretical predictions. (a) Experimental measured $\phi_{R}$ of $S$. cerevisiae along with the predictions (dashed lines) of our model. (b) The growth rate dependence of the correlation coefficients $I_{\chi, k}$ and $I_{\phi, \alpha}$. (c) The normalized enrichment score (NES) of GSEA of enriched gene sets. A positive NES of $k_{i}$-ordered GSEA means that the genes in the corresponding gene set are enriched in the regime of higher $k_{i}$. A positive NES of $\log _{2}$ FC-ordered GSEA means that the genes in the corresponding gene set are enriched in the regime of increasing $\chi_{i}$ when the nutrient changes from glucose to glycerol. (d) The enriched gene sets with their false discovery rate (FDR) q values of the single-sided permutation test. The higher the $-\log _{10}(\mathrm{FDR})$ value is, the more likely a gene set is enriched. (e) Summary of the multiple computed variables and parameters in the analysis of experimental data. Note that the effective mass of ribosomal proteins $m_{R}$ is calculated based on molecular weights of ribosomal proteins detected in the proteome (Appendix F). SC, synthetic complete medium. Glu, glucose. Gly, glycerol.

${ }_{419}$ lar, the main effect of heterogeneous translation speeds ${ }_{423}$ STM. The actual shape of the growth law curve depends 420 is making the growth law curve up-bent relative to the ${ }^{424}$ on two correlation coefficients: one is between the ribo${ }_{421}$ STM. The main effect of protein degradation is reduc- 425 some allocations and the translation speeds $\left(I_{\chi, k}\right)$; the 422 ing the slope and increasing the intercept relative to the ${ }_{426}$ other is between the protein mass fractions and protein 
a

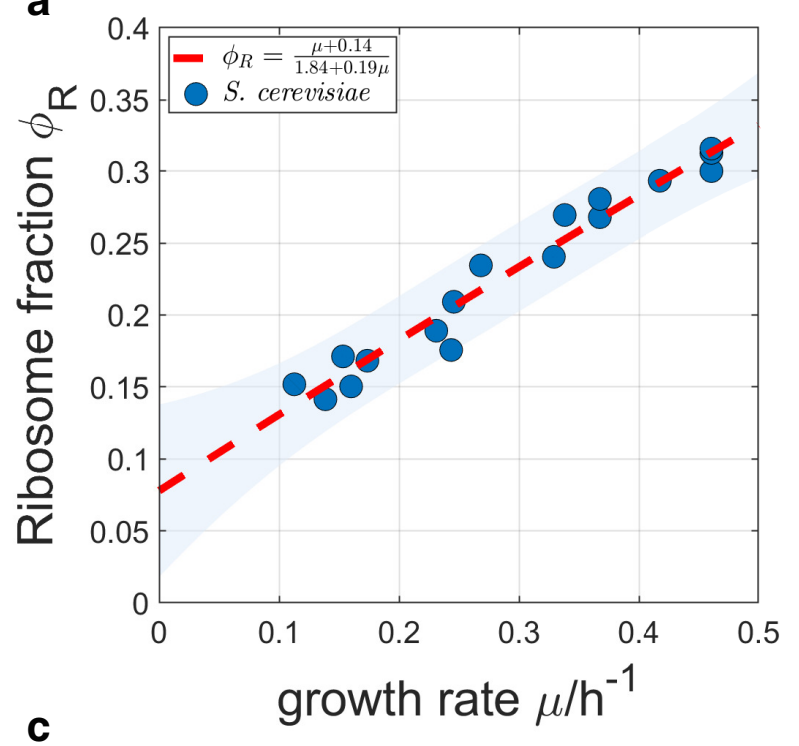

b

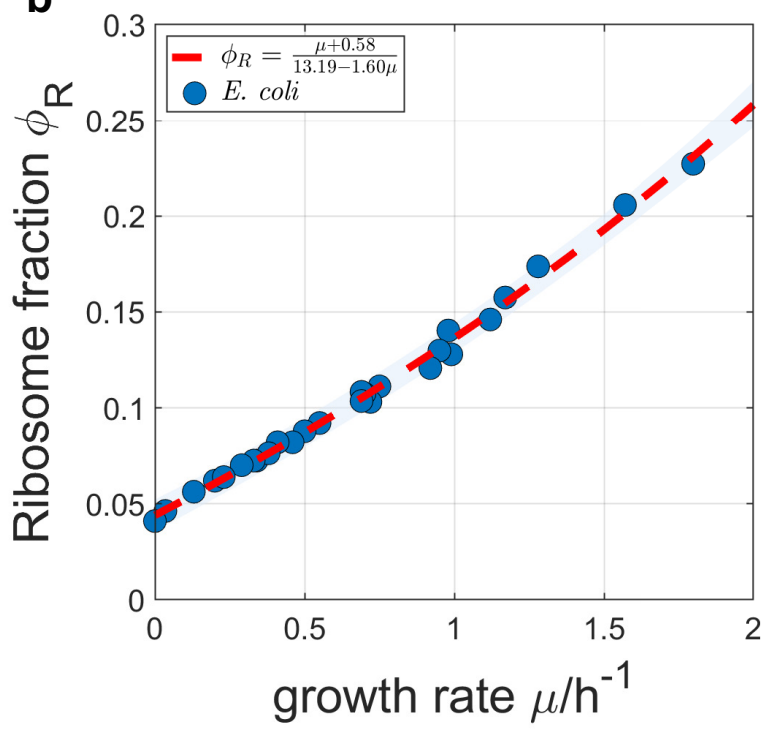

\begin{tabular}{|c|c|c|c|c|c|c|c|c|c|c|c|c|c|c|c|c|}
\hline \multirow[b]{2}{*}{ Organism } & \multicolumn{4}{|c|}{ Fitting parameters } & \multicolumn{3}{|c|}{ Known parameters } & \multicolumn{6}{|c|}{ Calculated values } & \multicolumn{2}{|c|}{ Reference values } & \multirow[b]{2}{*}{ Data source } \\
\hline & $\begin{array}{c}c_{1} \text { with } 95 \% \\
\text { confidence } \\
\text { interval } \\
(1 / \mathrm{h})\end{array}$ & $\begin{array}{c}\mathrm{c}_{2} \text { with } 95 \% \\
\text { confidence } \\
\text { interval }\end{array}$ & $\begin{array}{c}c_{3} \text { with } 95 \% \\
\text { confidence } \\
\text { interval } \\
(1 / \mathrm{h})\end{array}$ & RMSE & $\begin{array}{l}m_{R} \\
(\mathrm{Da})\end{array}$ & $\begin{array}{l}<\alpha>_{\phi} \\
(1 / h)\end{array}$ & $\begin{array}{c}a_{R} \\
(1 / h)\end{array}$ & $\phi_{0}$ & $\begin{array}{c}k_{R} \\
(\mathrm{Da} / \mathrm{min})\end{array}$ & $\left|\begin{array}{c}<k>x \\
(\mathrm{Da} / \mathrm{min})\end{array}\right|$ & $\begin{array}{c}\text { The range } \\
\text { of } \phi_{0}\end{array}$ & $\begin{array}{c}\text { The range } \\
\text { of } k_{R} \\
(\mathrm{Da} / \mathrm{min})\end{array}$ & $\begin{array}{c}\text { The range } \\
\text { of }<k>x \\
(\mathrm{Da} / \mathrm{min})\end{array} \mid$ & $\begin{array}{c}k_{R} \\
(\mathrm{Da} / \mathrm{min})\end{array}$ & $\langle\mathrm{k}\rangle_{\mathrm{X}}(\mathrm{Da} / \mathrm{min})$ & \\
\hline S. cerevisiae & $0.14 \pm 0.23$ & $0.19 \pm 2.28$ & $1.84 \pm 1.72$ & $1.35 \mathrm{e}-2$ & $1.40 \mathrm{e} 6$ & $4.42 \mathrm{e}-2$ & $2.90 \mathrm{e}-2$ & $5.45 e-2$ & $5.24 \mathrm{e} 4$ & $4.25 \mathrm{e} 4$ & $\begin{array}{c}-3.92 \\
- \\
9.93\end{array}$ & $\begin{array}{c}1.25 \mathrm{e} 3 \\
- \\
3.48 \mathrm{e} 12\end{array}$ & $\begin{array}{c}7.83 \mathrm{e} 2 \\
- \\
8.42 \mathrm{e} 4\end{array}$ & $\begin{array}{c}2.07 \mathrm{e} 4 \\
\text { Riba et al., } \\
2019\end{array}$ & \begin{tabular}{|c|}
$7.78 \mathrm{e} 4$ \\
- \\
$1.65 \mathrm{e} 5$ \\
\\
Riba et al., \\
2019 \\
Friedrich et al., \\
2021
\end{tabular} & $\begin{array}{c}\text { Metzl et al., } \\
2017\end{array}$ \\
\hline E.coli & $0.58 \pm 0.12$ & $-1.60 \pm 0.79$ & $13.19 \pm 1.74$ & $3.60 e-3$ & $8.07 e 5$ & 0 & 0 & $4.40 \mathrm{e}-2$ & $6.82 \mathrm{e} 4$ & $1.77 \mathrm{e} 5$ & $\begin{array}{c}3.10 \mathrm{e}-2 \\
- \\
6.10 \mathrm{e}-2\end{array}$ & $\begin{array}{c}4.54 \mathrm{e} 4 \\
- \\
1.11 \mathrm{e} 5\end{array}$ & $\begin{array}{c}1.54 \mathrm{e} 5 \\
- \\
2.01 \mathrm{e} 5\end{array}$ & $\begin{array}{r}\text { Order of mas } \\
\text { Arkin e } \\
\text { Gouy and G } \\
\text { Karpinets }\end{array}$ & $\begin{array}{l}\text { gnitude: } 10^{4}-10^{5} \\
\text { t.al., } 1998 \\
\text { rantham } 1980 \\
\text { et.al., } 2006\end{array}$ & $\begin{array}{l}\text { Dai et.al., } \\
2016\end{array}$ \\
\hline
\end{tabular}

FIG. 5. The full model fits different datasets. (a) The non-linear fitting to data from Ref. [6]. The shadow represents the $95 \%$ prediction interval. (b) The non-linear fitting to data from Ref. [7]. The shadow is the same as in (a). (c) Detailed fitting results of (a) and (b). Note that the reference value of $\langle k\rangle_{\chi}$ of (a) is approximated by $\langle k\rangle\left(1+I_{\chi, k}\right)$ where the range of $I_{\chi, k}$ can be found in Figure 4c.

${ }_{427}$ degradation rates $\left(I_{\phi, \alpha}\right)$. By analyzing the dataset from ${ }_{444}$ the ribosome profiling and mass spectrometry data from ${ }_{428}$ [14], we found that $I_{\phi, \alpha}$ is independent of growth rate, 445 [14]. In contrast, the fitting of $E$. coli data exhibits a ${ }_{429}$ while $I_{\chi, k}$ appears to be negatively correlated with the ${ }_{446}$ much smaller uncertainty, suggesting that common $I_{\chi, k}$ 430 growth rate. This means that cells tend to produce pro- 447 and $I_{\phi, \alpha}$ may apply to all the nutrient qualities used in ${ }_{431}$ teins with faster translation speeds in slow-growth con- 448 the experiments of Ref. [7]. This is to be tested when 432 ditions, which can be an economic strategy and under 449 genome-wide measurements, such as translation speeds, ${ }_{433}$ evolutionary selection. Remarkably, our theoretical pre- 450 of E. coli are available in the future.

${ }_{434}$ dictions of $\phi_{R}$ can reasonably match the experimentally 435 measured values [14], with a common fraction of inactive ${ }_{436}$ ribosomes $\phi_{0}$. Our results imply that the fraction of inac${ }_{437}$ tive ribosomes may be constant across different nutrient 438 qualities.

${ }^{451}$ We remark that in the absence of heterogeneous trans452 lation speeds and protein degradation, the mass fraction ${ }_{453}$ of protein $i, \phi_{i}$ must equal the ribosome allocation $\chi_{i}$. 454 Indeed, these two datasets are often highly correlated 455 among proteins in E. coli $[12,25]$. However, in our more ${ }_{439}$ We apply our model predictions to the growth law ${ }_{456}$ realistic models, $\phi_{i}$ depends on the translation speed ${ }_{440}$ curves of $S$. cerevisiae [6] and E. coli [7]. In the for- 457 and protein degradation rate. Given the same $\chi_{i}$, pro${ }_{441}$ mer case, the fitting of data to our model prediction is 458 teins with higher translation speeds or lower degradation ${ }_{442}$ subject to significant uncertainty. This agrees with the ${ }_{459}$ rates should have higher mass fractions (Appendix A). ${ }_{443}$ computed $I_{\chi, k}$ that are variable across conditions using ${ }_{460}$ We note that using the current genome-wide datasets of 
${ }_{461}$ S. cerevisiae, the predicted protein mass fractions $\phi_{i, p r e}{ }^{494}$ 462 based on the ribosome allocations $\chi_{i}$ [14], the translation ${ }_{463}$ speeds $k_{i}$ [9], and the protein degradation rates $\alpha_{i}[11]_{495}$ 464 do not correlate strong enough with the measured $\phi_{i}$ as ${ }_{465}$ expected. We note that these datasets are from different ${ }_{466}$ references, and the deviation is likely due to the noise in ${ }_{467}$ the measurements of $k_{i}$ (Supplementary Table S2). We ${ }_{468}$ expect our theories to be further verified when more ac469 curate measurements of translation speeds are available.

470 For simplicity, in this work, we assume that the trans471 lation speeds and protein degradation rates are invariant 472 as the nutrient quality changes. Therefore, we can use the ${ }_{473}$ two correlation coefficients $I_{\chi, k}$ and $I_{\phi, \alpha}$ to characterize a ${ }_{474}$ particular environment. We remark that our model can 475 be generalized to more complex scenarios in which the 476 translation speeds or protein degradation rates depend 477 on the growth rate [7]. In this case, one just needs to in478 clude four additional environmental-specific parameters: ${ }_{479} k_{R},\langle k\rangle, \alpha_{R}$, and $\langle\alpha\rangle$.

480

481

482 483 is

$$
\frac{d \phi_{i}}{d t}=\frac{\frac{d M_{i}}{d t} M-\frac{d M}{d t} M_{i}}{M^{2}}=\frac{\frac{d M_{i}}{d t}}{M}-\frac{\frac{d M}{d t}}{M} \frac{M_{i}}{M} .
$$

${ }_{484}$ In the steady state, $\phi_{i}$ doesn't change so that Eq. (13) ${ }_{507}$ Combining Eq. (21) and Eq. (22) allows us to solve $\phi_{R}$ ${ }_{485}$ equals 0 . Combined with the definition of growth rate 508 as a function of $\mu$ and we obtain Eq. (12) 486 and Eq. (1), we obtain

$$
\frac{d \phi_{i}}{d t}=\frac{k_{i} \chi_{i}\left(\phi_{R}-\phi_{0}\right)}{m_{R}}-\alpha_{i} \phi_{i}-\mu \phi_{i}=0,
$$

${ }_{487}$ which leads to Eq. (2). In the steady state, we can write ${ }_{488} \phi_{i}$ using Eq. (2) as

$$
\phi_{i}=\frac{k_{i} \chi_{i} /\left(\mu+\alpha_{i}\right)}{\sum_{j} k_{j} \chi_{j} /\left(\mu+\alpha_{j}\right)} .
$$

489

We can also rewrite Eq. (2) using $\sum_{i} \phi_{i}=1$ as

$$
1=\frac{\phi_{R}-\phi_{0}}{m_{R}} \sum_{i} \frac{k_{i} \chi_{i}}{\left(\mu+\alpha_{i}\right)}
$$

490

\section{B. Derivation of Equation (3)}

491 We rewrite Eq. (2) as

$$
m_{R} \mu \phi_{i}+m_{R} \alpha_{i} \phi_{i}=k_{i} \chi_{i}\left(\phi_{R}-\phi_{0}\right) .
$$

${ }_{492}$ We then sum up for all proteins and obtain

$$
m_{R} \mu+m_{R} \sum_{i=1}^{n} \alpha_{i} \phi_{i}=\left(\phi_{R}-\phi_{0}\right) \sum_{i=1}^{n} k_{i} \chi_{i},
$$

${ }_{493}$ which leads to Eq. (3).

\section{Derivation of Equation (4)}

In deriving Eq. (4), we neglect protein degradation 496 and rewrite Eq. (3) as

$$
\phi_{R}=\frac{m_{R} \mu}{k_{R} \chi_{R}+\left(1-\chi_{R}\right)\langle k\rangle_{\chi}}+\phi_{0} .
$$

${ }_{497}$ Meanwhile, we compute the growth rate using the auto498 catalytic nature of ribosomal proteins,

$$
\mu=\frac{\frac{d M_{R}}{d t}}{M_{R}}=\frac{k_{R} \chi_{R}}{m_{R}}\left(1-\frac{\phi_{0}}{\phi_{R}}\right) .
$$

${ }_{499}$ The above equation allows us to replace $\chi_{R}$ by $\mu$ in Eq. 500 (19), from which we obtain Eq. (4).

\section{$501 \quad$ D. Derivation of the full model}

502 In this section we derive the full model considering 503 both the heterogeneities in the translation speeds and ${ }_{504}$ protein degradation rates. We rewrite Eq. (3) in the 505 main text as

$$
\phi_{R}=\frac{m_{R}\left[\mu+\alpha_{R} \phi_{R}+\left(1-\phi_{R}\right)\langle\alpha\rangle_{\phi}\right]}{k_{R} \chi_{R}+\left(1-\chi_{R}\right)\langle k\rangle_{\chi}}+\phi_{0} .
$$

${ }_{506}$ Meanwhile, the growth rate is

$$
\mu=\frac{k_{R} \chi_{R}}{m_{R}}\left(1-\frac{\phi_{0}}{\phi_{R}}\right)-\alpha_{R} .
$$

$$
\phi_{R}=\frac{\mu+c_{1}}{c_{2} \mu+c_{3}},
$$

509 where

$$
\begin{gathered}
c_{1}=\frac{\langle k\rangle_{\chi} \phi_{0}}{m_{R}}+\langle\alpha\rangle_{\phi}, \\
c_{2}=1-\frac{\langle k\rangle_{\chi}}{k_{R}}, \\
c_{3}=\langle\alpha\rangle_{\phi}-\frac{\alpha_{R}\langle k\rangle_{\chi}}{k_{R}}+\frac{\langle k\rangle_{\chi}}{m_{R}} .
\end{gathered}
$$

It is straightforward to find that the condition for Eq. (12) to be monotonically increasing is that $c_{3}>c_{1} c_{2}$. Using the above expressions, we find that

$$
\begin{aligned}
& c_{3}-c_{1} c_{2}=\frac{\langle k\rangle_{\chi}\left(1-\phi_{0}\right)}{m_{R}}+ \\
& \frac{\langle k\rangle_{\chi}^{2} \phi_{0}}{k_{R} m_{R}}+\frac{\langle k\rangle_{\chi}\left(\langle\alpha\rangle_{\phi}-\alpha_{R}\right)}{k_{R}} .
\end{aligned}
$$

510 We find that the first two terms are always positive, and 511 the last term is positive as long as $I_{\alpha, \phi}$ is not too close to $512-1$. Therefore, the $\phi_{R}(\mu)$ curve must be monotonically 513 increasing. It is straightforward to find that the second ${ }_{514}$ derivative of the $\phi_{R}(\mu)$ curve is proportional to $\left(c_{1} c_{2}-\right.$ $\left.{ }_{515} c_{3}\right) c_{2}$, which is always positive as long as $I_{\chi, k}$ is not too ${ }_{516}$ close to -1 . 


\section{E. Details of the numerical simulations}

518

summarize the parameters we use in the numerical 519 simulations in Supplementary Table S1. We consider a 5 ${ }_{520}$ cell with 4000 genes. We set the elongation speed $k_{i}$ and ${ }_{521}$ the degradation rates $\alpha_{i}$ of non-ribosomal genes to follow ${ }_{522}$ lognormal distributions. We set $k_{R}=2.07 \times 10^{4} \mathrm{Da} / \mathrm{min}$, ${ }_{523}\langle k\rangle=4.80 \times 10^{4} \mathrm{Da} / \mathrm{min}, \alpha_{R}=4.83 \times 10-4 \mathrm{~min}^{-1}$, and ${ }_{524}\langle\alpha\rangle=1.10 \times 10^{-3} \mathrm{~min}^{-1}$ as the experimentally measured ${ }_{525}$ values of $S$. cerevisiae $[9,11]$. The coefficients of vari${ }_{526}$ ation $(\mathrm{CV})$ of the lognormal distributions can be found ${ }_{527}$ in Supplementary Table S1. In all simulations, we set ${ }_{528} \phi_{0}=0.08$. We note that in Figure $2 \mathrm{a}$, we set $\alpha_{i}=0$ ${ }_{529}$ for all proteins and in Figure $2 \mathrm{~b}$, we set $k_{i}=\langle k\rangle$ for all ${ }_{530}$ proteins. We note that for given $I_{\chi, k}$ and $I_{\phi, \alpha}, k_{i}$ and $\alpha_{i}$ 531 are fixed for environments with different $\chi_{i}$.

${ }_{532}$ To simulate a random environment, we generate a ran${ }_{533}$ dom $\chi_{R}$. Meanwhile, a lognormal distribution of $\chi_{i}$ of ${ }_{534}$ non-ribosomal genes is also randomly generated. The ${ }_{535} \mathrm{CV}$ of the lognormal distribution is included in Supple${ }_{536}$ mentary Table S1. We then search for the $\phi_{R}$ and $\mu$ that ${ }_{537}$ simultaneously satisfy Eq. (22) and Eq. (16). $\phi_{i}, I_{\chi, k}$ ${ }_{538}$ and $I_{\phi, \alpha}$ are then calculated using Eq. (3), Eq. (7) and ${ }_{539}$ Eq. (11), respectively. For a chosen pair of $I_{\chi, k}$ and $I_{\phi, \alpha}$, ${ }_{540}$ the predicted $\phi_{R}(\mu)$ curve is obtained using Eq. (12).

${ }_{541}$ To obtain Figure. 2d, g, we randomly sample 20 points ${ }_{542}$ from Fig. 2c, f respectively, fit them using Eq. (12), ${ }_{543}$ and calculate the resulting RMSE. We repeat the above 5 ${ }_{544}$ process 5000 times.

\section{F. Details of the experimental data analysis}

${ }_{546}$ For the ribosome profiling data [14], we first trim the ${ }_{547}$ adapter with Cutadapt (version 3.4) [26]. Then we use 6 ${ }_{548}$ Bowtie2 (version 2.4.2) [27] to eliminate ribosomal RNAs ${ }_{549}$ (rRNA) as mentioned in [28]. The cleaned reads are then ${ }_{605}$ 550 mapped to $S$. cerevisiae genome R64.1.1 with HISAT2 551 (version 2.2.1) [29]. Read counts are then generated with 552 featureCount (version 2.0.1) [30]. The ribosome alloca${ }_{553}$ tion $\chi_{i}$ is calculated based on the count fraction.

${ }_{554}$ For the proteomics data [14], we perform the absolute

${ }_{555}$ quantification (or the in-sample relative quantification) of ${ }_{611} \quad$ For GSEA analysis, we first perform the differential ex556 proteins based on the intensities of peptides using xTop ${ }_{612}$ pression analysis on the ribosome profiling data of WT or ${ }_{557}$ (version 1.2) [12]. The intensity ratio of 2 proteins in the ${ }_{613} \Delta$ Naa10 cells using the package DEseq2 (version 1.24.0) 558 same sample of proteomics data does not directly rep- 614 [33] in $\mathrm{R}$ (version 3.6.1). The $\log _{2}$ fold changes of counts 559 resent the real abundance (either the mass or the copy 615 when cells changed from $\mathrm{SC}+2 \%$ glucose to SC+2\% glyc560 number) ratio so that the abundance fraction can not be ${ }_{616}$ erol as well as the FDR q values are calculated. Riboso${ }_{561}$ replaced with the intensity fraction $[12,31]$. XTop is a 617 mal genes and genes with FDR q value $>0.05$ are elimi562 novel software that accurately calculates the in-sample ${ }_{618}$ nated. We then pick out genes that also exist in the data ${ }_{563}$ relative protein copy number with the maximum a pos- ${ }_{619}$ sets of $k_{i}$. GSEA on these genes are then performed twice ${ }_{564}$ teriori probability (MAP) algorithm [12]. We then calcu- 620 using the R package clusterProfiler (version 3.12.0) [34] ${ }_{565}$ late all proteins' mass fraction $\phi_{i}$ with the xTop results ${ }_{621}$ and org.Sc.sgd.db (version 3.8.2) [35]. In the first GSEA, 566 and the protein molecular mass. In [12], the authors fur- 622 genes are ordered by the $\log _{2}$ fold change (denoted as ${ }_{567}$ ther calibrated $\phi_{i}$ with ribosome profiling data assuming $623 \log _{2}$ FC-ordered GSEA). In the second GSEA, genes are 568 homogeneous $k_{i}$. In this work, we alternatively calibrate 624 ordered by $k_{i}$ (denoted as $k_{i}$-ordered GSEA). We then

${ }_{569} \phi_{i}$ with $L^{-0.57}$ where $L$ is the protein length, as men571 ribosome profiling data, although it reduces the distance 57 between $\chi_{i}$ and calibrated $\phi_{i}[12]$. We also show the 574 Supplementary Figure S1b, c. To compute $\phi_{R}$, we sum up the $\phi_{i}$ of all proteins annotated as the cytoplasmic 577 (SGD).

579 mentioned in [9]. $k_{i}$ is then calculated using the rela80 tionship $k_{i}=v_{i} a_{i}$. For the degradation rate $\alpha_{i}$, data is ${ }_{82} I_{\phi, \alpha},\langle k\rangle$ and $\langle\alpha\rangle$ for non-ribosomal genes that exist in ${ }_{583}$ all data sets of $\chi_{i}, \phi_{i}, k_{i}$ and $\alpha_{i}$. We also calculate the ${ }_{54} \chi$-averaged $k$ of ribosomal proteins as $k_{R}$ and $\phi$-averaged For the molecular mass of the ribosome, we calculate ${ }_{587}$ the effective $m_{R}$. Considering the efficiency of the mass ${ }_{589}$ Spectrometry (MS), not all proteins can be detected. 590 weights of ribosomal proteins detected in the proteome. ${ }_{591}$ Because most of the ribosomal proteins can be expressed
59 two paragous genes in $S$. cerevisiae, we count the av${ }_{593}$ erage molecular mass when both proteins of the paralogs tions of $\phi_{R}$ using the real ribosome mass $\left(m_{R}=1.40 e 6\right.$ Da) in Supplementary Figure S1a.

598 curve, $\mathrm{OD}_{600}$ versus time with the method mentioned in 599 [32]. Briefly, the slopes of $\ln \left(\mathrm{OD}_{600}\right)$ versus time in 5600 point windows are calculated. Then windows with slopes 601 that are at least $95 \%$ of the maximum slope are extracted. ${ }_{03}$ as the growth rate. With these results, we predict the 604 corresponding $\phi_{R}(\mu)$ curves and compare them with the experimental data points.

${ }_{07}$ of non-ribosomal proteins with Eq. (15). Pearson corre608 lation coefficients $\rho$ between $\phi_{i, p r e}$ and $\phi_{i}$ are calculated. ${ }_{609}$ We also compute $\rho$ under the assumptions that $\alpha_{i}=0$ $k_{i}=\langle k\rangle$ (Supplementary Table S2). 
625 find the common gene sets from GO database [17, 18] ${ }_{639}$ ${ }_{626}$ enriched in these two GSEA. The cut-off criteria are set ${ }_{627}$ as the $\mathrm{p}$ value $<0.05$ and the FDR $\mathrm{q}$ value $<0.25$. The 628 number of permutations used in the analysis is $1 \mathrm{e} 5$.

\section{G. Details of fitting in Figure 5}

\section{H. A summary of the variables used in this work}

\section{Variables Meaning}

$N \quad$ number of genes

$M_{i} \quad$ mass of protein $i$

$M \quad$ total mass of all proteins

$k_{i} \quad$ the mass of translated protein $i$ per unit time

$k_{R} \quad$ the mass of translated ribosomal protein per unit time

$\langle k\rangle \quad$ the arithmetic average mass of translated protein mass over non-ribosomal proteins per unit time

$\langle k\rangle_{\chi} \quad$ the $\chi$-weighted average mass of translated non-ribosomal proteins per unit time

$\chi_{i} \quad$ the fraction of active ribosomes producing protein $i$ in the pool of total active ribosomes

$\chi_{R} \quad$ the fraction of active ribosomes producing themselves in the pool of total active ribosomes

$\tilde{\chi}_{i} \quad$ the fraction of active ribosomes producing protein $i$ in the pool of active ribosomes translating non-ribosomal proteins

$R \quad$ total number of ribosomes

$R_{0} \quad$ total number of inactive ribosomes

$\alpha_{i} \quad$ degradation rate of protein $i$

$\alpha_{R} \quad$ degradation rate of the ribosomal protein

$\langle\alpha\rangle \quad$ the arithmetic average degradation rate over non-ribosomal proteins

$\langle\alpha\rangle_{\phi} \quad$ the $\phi$-weighted average degradation rate over non-ribosomal proteins

$\phi_{i} \quad$ the mass fraction of protein $i$

$\phi_{R} \quad$ the mass fraction of ribosomes

$\phi_{0} \quad$ the mass fraction of inactive ribosomes

$\widetilde{\phi}_{i} \quad$ the mass fraction of non-ribosomal protein $i$ in the pool of all non-ribosomal proteins

$m_{R} \quad$ molecular mass of ribosome

$\mu \quad$ the growth rate

$I_{\chi, k} \quad$ the metric quantifying the correlation between the ribosome allocations and the translation speeds of non-ribosomal proteins

$I_{\phi, \alpha} \quad$ the metric quantifying the correlation between the mass fractions and the degradation rates of non-ribosomal proteins
${ }_{630}$ Nonlinear fitting is performed with MATLAB (version $\left.{ }_{631} \mathrm{R} 2020 \mathrm{~b}\right)$. We obtain the fitting parameters $c_{1}, c_{2}$ and ${ }_{642}$ ${ }_{632} c_{3}$ with their $95 \%$ confidence intervals, and then com- ${ }_{643}$ ${ }_{633}$ pute $\phi_{0}, k_{R}$ and $\langle k\rangle_{\chi}$ using Eqs. $(23,24,25)$. To com- ${ }^{644}$ ${ }_{634}$ pute the ranges of these values, we numerically find the ${ }^{645}$ ${ }_{635}$ maximum and the minimum value of the multivariate ${ }_{647}^{646}$ ${ }_{636}$ functions $\phi_{0}\left(c_{1}, c_{2}, c_{3}\right), k_{R}\left(c_{1}, c_{2}, c_{3}\right)$ and $\langle k\rangle_{\chi}\left(c_{1}, c_{2}, c_{3}\right)^{648}$ ${ }_{637}$ as their upper and lower bounds, where the ranges of $c_{1},{ }_{649}$ ${ }_{638} c_{2}$ and $c_{3}$ are their $95 \%$ confidence intervals.
[1] F. C. Neidhardt and B. Magasanik, Studies on the role of ribonucleic acid in the growth of bacteria, Biochimica et biophysica acta 42, 99 (1960).

[2] O. Maaløe, Regulation of the protein-synthesizing machinery - ribosomes, trna, factors, and so on, in Biological regulation and development (Springer, 1979) pp. 487-542.

[3] H. Bremer and P. P. Dennis, Modulation of chemical composition and other parameters of the cell at different exponential growth rates, EcoSal Plus 3 (2008). 
[4] M. Scott, C. W. Gunderson, E. M. Mateescu, Z. Zhang, 715 and T. Hwa, Interdependence of Cell Growth and Gene 716 Expression: Origins and Consequences, Science 330, 717 1099 (2010).

[5] S. Hui, J. M. Silverman, S. S. Chen, D. W. Erickson, 719 M. Basan, J. Wang, T. Hwa, and J. R. Williamson, Quan- 720 titative proteomic analysis reveals a simple strategy of 721 global resource allocation in bacteria, Molecular Systems 722 Biology 11, 784 (2015).

[6] E. Metzl-Raz, M. Kafri, G. Yaakov, I. Soifer, Y. Gurvich, 724 and N. Barkai, Principles of cellular resource allocation 725 revealed by condition-dependent proteome profiling, Elife 726 6, e28034 (2017).

[7] X. Dai, M. Zhu, M. Warren, R. Balakrishnan, V. Patsalo, 728 H. Okano, J. R. Williamson, K. Fredrick, Y.-P. Wang, 729 and T. Hwa, Reduction of translating ribosomes enables 730 escherichia coli to maintain elongation rates during slow 731 growth, Nature microbiology 2, 1 (2016).

[8] S. Klumpp, J. Dong, and T. Hwa, On ribosome load, 733 codon bias and protein abundance, PLoS ONE 7, e48542 734 (2012).

[9] A. Riba, N. Di Nanni, N. Mittal, E. Arhné, A. Schmidt, 736 and M. Zavolan, Protein synthesis rates and ribosome 737 occupancies reveal determinants of translation elongation 738 rates, Proceedings of the national academy of sciences 739 116, 15023 (2019).

10] O. Gefen, O. Fridman, I. Ronin, and N. Q. Balaban, Di- 741 rect observation of single stationary-phase bacteria re- 742 veals a surprisingly long period of constant protein pro- 743 duction activity, Proceedings of the National Academy of 744 Sciences 111, 556 (2014).

11] P.-J. Lahtvee, B. J. Sánchez, A. Smialowska, S. Kas- 740 vandik, I. E. Elsemman, F. Gatto, and J. Nielsen, Ab- 747 solute quantification of protein and mrna abundances 748 demonstrate variability in gene-specific translation effi- 749 ciency in yeast, Cell Systems 4, 495 (2017).

12] M. Mori, Z. Zhang, A. Banaei-Esfahani, J.-B. Lalanne, 751 H. Okano, B. C. Collins, A. Schmidt, O. T. Schubert, D.- 752 S. Lee, G.-W. Li, R. Aebersold, T. Hwa, and C. Ludwig, 753 From coarse to fine: the absolute escherichia coli pro- 754 teome under diverse growth conditions, Molecular Sys- 755 tems Biology 17, e9536 (2021).

13] G.-W. Li, D. Burkhardt, C. Gross, and J. S. Weiss- 757 man, Quantifying absolute protein synthesis rates reveals 758 principles underlying allocation of cellular resources, Cell 759 157, 624 (2014).

14] U. A. Friedrich, M. Zedan, B. Hessling, K. Fenzl, 761 L. Gillet, J. Barry, M. Knop, G. Kramer, and B. Bukau, 762 $\mathrm{N} \alpha$-terminal acetylation of proteins by nata and natb 763 serves distinct physiological roles in saccharomyces cere- 764 visiae, Cell Reports 34, 108711 (2021).

[15] A. Subramanian, P. Tamayo, V. K. Mootha, S. Mukher- 766 jee, B. L. Ebert, M. A. Gillette, A. Paulovich, S. L. ${ }_{767}$ Pomeroy, T. R. Golub, E. S. Lander, and J. P. Mesirov, 768 Gene set enrichment analysis: A knowledge-based ap- 769 proach for interpreting genome-wide expression profiles, 770 Proceedings of the National Academy of Sciences 102, 771 15545 (2005).

6] V. K. Mootha, C. M. Lindgren, K.-F. Eriksson, A. Sub- 773 ramanian, S. Sihag, J. Lehar, P. Puigserver, E. Carls- 774 son, M. Ridderstråle, E. Laurila, and et al., Pgc-1alpha- 775 responsive genes involved in oxidative phosphorylation 776 are coordinately downregulated in human diabetes, Na- 777 ture Genetics 34, 267-273 (2003).
[17] M. Ashburner, C. A. Ball, J. A. Blake, D. Botstein, H. Butler, J. M. Cherry, A. P. Davis, K. Dolinski, S. S. Dwight, J. T. Eppig, M. A. Harris, D. P. Hill, L. IsselTarver, A. Kasarskis, S. Lewis, J. C. Matese, J. E. Richardson, M. Ringwald, G. M. Rubin, and G. Sherlock, Gene ontology: tool for the unification of biology, Nature Genetics 25, 25 (2000).

[18] S. Carbon, E. Douglass, B. M. Good, D. R. Unni, N. L. Harris, C. J. Mungall, S. Basu, R. L. Chisholm, R. J. Dodson, E. Hartline, P. Fey, P. D. Thomas, L.-P. Albou, D. Ebert, M. J. Kesling, H. Mi, A. Muruganujan, X. Huang, T. Mushayahama, S. A. LaBonte, D. A. Siegele, G. Antonazzo, H. Attrill, N. H. Brown, P. Garapati, S. J. Marygold, V. Trovisco, G. dos Santos, K. Falls, C. Tabone, P. Zhou, J. L. Goodman, V. B. Strelets, J. Thurmond, P. Garmiri, R. Ishtiaq, M. RodríguezLópez, M. L. Acencio, M. Kuiper, A. Lægreid, C. Logie, R. C. Lovering, B. Kramarz, S. C. C. Saverimuttu, S. M. Pinheiro, H. Gunn, R. Su, K. E. Thurlow, M. Chibucos, M. Giglio, S. Nadendla, J. Munro, R. Jackson, M. J. Duesbury, N. Del-Toro, B. H. M. Meldal, K. Paneerselvam, L. Perfetto, P. Porras, S. Orchard, A. Shrivastava, H.-Y. Chang, R. D. Finn, A. L. Mitchell, N. D. Rawlings, L. Richardson, A. Sangrador-Vegas, J. A. Blake, K. R. Christie, M. E. Dolan, H. J. Drabkin, D. P. Hill, L. Ni, D. M. Sitnikov, M. A. Harris, S. G. Oliver, K. Rutherford, V. Wood, J. Hayles, J. Bähler, E. R. Bolton, J. L. D. Pons, M. R. Dwinell, G. T. Hayman, M. L. Kaldunski, A. E. Kwitek, S. J. F. Laulederkind, C. Plasterer, M. A. Tutaj, M. Vedi, S.J. Wang, P. D'Eustachio, L. Matthews, J. P. Balhoff, S. A. Aleksander, M. J. Alexander, J. M. Cherry, S. R. Engel, F. Gondwe, K. Karra, S. R. Miyasato, R. S. Nash, M. Simison, M. S. Skrzypek, S. Weng, E. D. Wong, M. Feuermann, P. Gaudet, A. Morgat, E. Bakker, T. Z. Berardini, L. Reiser, S. Subramaniam, E. Huala, C. N. Arighi, A. Auchincloss, K. Axelsen, G. ArgoudPuy, A. Bateman, M.-C. Blatter, E. Boutet, E. Bowler, L. Breuza, A. Bridge, R. Britto, H. Bye-A-Jee, C. C. Casas, E. Coudert, P. Denny, A. Estreicher, M. L. Famiglietti, G. Georghiou, A. Gos, N. Gruaz-Gumowski, E. Hatton-Ellis, C. Hulo, A. Ignatchenko, F. Jungo, K. Laiho, P. L. Mercier, D. Lieberherr, A. Lock, Y. Lussi, A. MacDougall, M. Magrane, M. J. Martin, P. Masson, D. A. Natale, N. Hyka-Nouspikel, S. Orchard, I. Pedruzzi, L. Pourcel, S. Poux, S. Pundir, C. Rivoire, E. Speretta, S. Sundaram, N. Tyagi, K. Warner, R. Zaru, C. H. Wu, A. D. Diehl, J. N. Chan, C. Grove, R. Y. N. Lee, H.M. Muller, D. Raciti, K. V. Auken, P. W. Sternberg, M. Berriman, M. Paulini, K. Howe, S. Gao, A. Wright, L. Stein, D. G. Howe, S. Toro, M. Westerfield, P. Jaiswal, L. Cooper, and J. Elser, The gene ontology resource: enriching a GOld mine, Nucleic Acids Research 49, D325 (2020).

[19] J. Gutin, A. Sadeh, A. Rahat, A. Aharoni, and N. Friedman, Condition-specific genetic interaction maps reveal crosstalk between the camp/pka and the hog mapk pathways in the activation of the general stress response, Molecular Systems Biology 11, 829 (2015).

[20] D. W. Erickson, S. J. Schink, V. Patsalo, J. R. Williamson, U. Gerland, and T. Hwa, A global resource allocation strategy governs growth transition kinetics of escherichia coli, 551, 119 (2017). 
778 [21] A. L. Goldberg and A. C. S. John, Intracellular protein 802 779 degradation in mammalian and bacterial cells: Part 2, 803 $780 \quad$ 45, 747 (1976).

781 [22] A. Arkin, J. Ross, and H. H. McAdams, Stochastic ki- 805 netic analysis of developmental pathway bifurcation in 806 phage lambda-infected escherichia coli cells, 149, 1633807 (1998).

[23] M. Gouy and R. Grantham, Polypeptide elongation and 809 trna cycling in escherichia coli: a dynamic approach, 810 FEBS letters 115, 151 (1980).

$24]$ T. V. Karpinets, D. J. Greenwood, C. E. Sams, and J. T. ${ }_{812}^{812}$ Ammons, RNA:protein ratio of the unicellular organ- 813 ism as a characteristic of phosphorous and nitrogen sto- 814 ichiometry and of the cellular requirement of ribosomes 815 for protein synthesis 4, 10.1186/1741-7007-4-30 (2006). $\quad 816$

25] T.-Y. Liu, H. H. Huang, D. Wheeler, Y. Xu, J. A. Wells, 817 Y. S. Song, and A. P. Wiita, Time-resolved proteomics 818 extends ribosome profiling-based measurements of pro- 819 tein synthesis dynamics, Cell Systems 4, 636 (2017).

820 M. Martin, Cutadapt removes adapter sequences from
high-throughput sequencing reads, EMBnet.journal 17, 10 (2011).

27] B. Langmead and S. L. Salzberg, Fast gapped-read align- ${ }_{824}^{823}$ ment with bowtie 2, Nature methods $\mathbf{9}, 357$ (2012).
[28] C. . V. Galmozzi, D. Merker, U. A. Friedrich, K. Döring, and G. Kramer, Selective ribosome profiling to study interactions of translating ribosomes in yeast, Nature protocols 14, 2279 (2019).
[29] D. Kim, J. M. Paggi, C. Park, C. Bennett, and S. L. Salzberg, Graph-based genome alignment and genotyping with hisat2 and hisat-genotype, Nature biotechnology 37, 907 (2019).

[30] Y. Liao, G. K. Smyth, and W. Shi, featureCounts: an efficient general purpose program for assigning sequence reads to genomic features, Bioinformatics 30, 923 (2013).

[31] F. Calderón-Celis, J. R. Encinar, and A. Sanz-Medel, Standardization approaches in absolute quantitative proteomics with mass spectrometry, Mass spectrometry reviews 37, 715 (2018).

[32] B. G. Hall, H. Acar, A. Nandipati, and M. Barlow, Growth Rates Made Easy, Molecular Biology and Evolution 31, 232 (2013).

[33] M. I. Love, W. Huber, and S. Anders, Moderated estimation of fold change and dispersion for rna-seq data with deseq2, Genome Biology 15, 550 (2014).

823 [34] G. Yu, L.-G. Wang, Y. Han, and Q.-Y. He, clusterProfiler: an $\mathrm{r}$ package for comparing biological themes among gene clusters, OMICS: A Journal of Integrative Biology 16, 284 (2012).

827 [35] M. Carlson, org.sc.sgd.db: Genome wide annotation for yeast, (2019), r package version 3.8.2. 